\title{
Functional Status in Behavioral Variant Frontotemporal Dementia: A Systematic Review
}

\author{
Thais Bento Lima-Silva, ${ }^{1}$ Valéria Santoro Bahia, ${ }^{1}$ \\ Ricardo Nitrini, ${ }^{1}$ and Mônica Sanches Yassuda ${ }^{1,2}$ \\ ${ }^{1}$ Neurology Department, University of São Paulo School of Medicine, Brazil \\ ${ }^{2}$ Gerontology Department, School of Arts, Sciences and Humanities of the University of São Paulo, \\ Avenida Dr. Eneas de Carvalho Aguiar 255, 05403-900 São Paulo, SP, Brazil
}

Correspondence should be addressed to Mônica Sanches Yassuda; yassuda@usp.br

Received 14 April 2013; Accepted 20 September 2013

Academic Editor: Cristiano Capurso

Copyright (C) 2013 Thais Bento Lima-Silva et al. This is an open access article distributed under the Creative Commons Attribution License, which permits unrestricted use, distribution, and reproduction in any medium, provided the original work is properly cited.

\begin{abstract}
The aim was to conduct a systematic review of studies that described the functional profile of patients with behavioral variant frontotemporal dementia (bvFTD), published between 2000 and 2013. The bibliographic search was conducted using the terms "frontotemporal dementia" and "frontotemporal lobar degeneration" in combination with "independence," "functionality," "basic activities of daily living," "disability," and scales that measure functional performance: "Disability Assessment for Dementia-DAD," "Functional Activities Questionnaire (FAQ)," "Direct Assessment of Functional Status (DAFS)." To be included in the review, the study had to mention the characterization of the functional status of patients with bvFTD in the objectives of the study, using a previously validated instrument of functional assessment. Fourteen studies met this criterion. The reviewed studies suggested that individuals with bvFTD have greater functional impairment when compared to those with other subtypes of frontotemporal lobar degeneration or Alzheimer's disease. The studies documented a significant association between cognitive impairment and measures of functionality in these patients. The cognitive profile of patients may predict faster functional decline.
\end{abstract}

\section{Introduction}

bvFTD is a clinical syndrome characterized by progressive impairment in behavior and personality as well as social, cognitive, and functional abilities, which predominantly affects middle-aged adults $[1,2]$. These changes stem from frontotemporal lobar degeneration (FTLD) secondary to a spectrum of heterogeneous pathologies [3, 4]. Despite recent advances in characterizing bvFTD, diagnosing this syndrome remains challenging. Patients may be erroneously considered to be cognitively unimpaired, while others are diagnosed with psychiatric disorders or Alzheimer's disease (AD) [5].

In 2011, a set of revised diagnostic criteria was proposed for the bvFTD based on recent publications and a consortium of international research groups [6]. The revised criteria were validated through the retrospective review of clinical records and the comparison of the accuracy of original and revised criteria in a sample of patients from 16 brain banks with autopsy-confirmed FTLD. With the revised criteria, a diagnosis of "possible" bvFTD requires three of the six clinically discriminated characteristics (loss of inhibition, apathy/inertia, loss of empathy, perseveration/compulsive behaviors, hyperorality, and dysexecutive neuropsychological profile). "Probable" bvFTD requires the additional features of functional disability and characteristic neuroimaging, whereas bvFTD "with definitive frontotemporal lobar degeneration" requires histopathological conformation or confirmation of pathogenic mutation. The new criteria are expected to contribute by allowing earlier diagnosis of the syndrome and differential diagnosis with psychiatric disorders.

There is a consensus among researchers of bvFTD that, in the absence of specific biomarkers, investigating functional status is essential for the diagnosis and treatment of the syndrome, given that the impact on the activities of daily living 
can be used as a diagnostic parameter [6]. Yet, studies investigating the functional performance of patients with FTLD (bvFTD and other subtypes such as nonfluent progressive aphasia-NFPA and semantic dementia-SD) are scarce [2].

New studies characterizing the functional profile of patients with bvFTD are warranted as they can contribute to the diagnostic process and rehabilitation of patients. Moreover, functional dependence in bvFTD creates burden on caregivers and family members and generates additional social costs. Functional rehabilitation and compensation may alleviate some of these problems. Therefore, the aim of the present investigation was to carry out a systematic review of studies describing the functional status of patients diagnosed with bvFTD, published between 2000 and 2013.

\section{Methods}

This review is based on a literature search conducted in September 2013. The bibliographic search was carried out in Medline, PsycInfo, PubMed, LILAC's, SciELO, and ScienceDirect databases using the search term "frontotemporal dementia" and "frontotemporal lobar degeneration" in combination with "functionality", "independence", "basic activities of daily living", "disability", and names of scales that measure functional performance: "Disability Assessment for Dementia-DAD", "Functional Activities Questionnaire (FAQ)", and "Direct Assessment of Functional Status (DAFS)", for retrieval of studies published between 2000 and 2013.

A total of 119 studies were retrieved, including articles, theses, dissertations, and book chapters. For inclusion in the review, studies had to cite in the objectives the characterization of the functional status of patients with bvFTD, using a previously validated functional assessment instrument. A total of 14 clinical studies met this criterion.

\section{Results}

This review identified a limited number of studies which addressed the issue of functionality in patients with variants of FTLD using validated instruments. Among these studies, a small number included in their sample a significant number of patients with bvFTD. We found two research studies that addressed the correlation between functional performance and executive dysfunction in patients with bvFTD, when compared to other dementia cases $[7,8]$. Two studies investigated functional performance compared to overall cognitive performance in patients with different dementia subtypes $[9,10]$. Five studies compared functional performance among patients with various subtypes of FTLD [11-15] one study linked functional performance to emotional aspects [16], another correlated functional decline in bvFTD patients with caregiver burden [17], and finally, some studies compared the performance of patients with dementia in direct versus indirect assessments of functionality $[8,18,19]$. Two studies investigated rate of functional change $[2,10]$.

The reviewed studies suggested that individuals with bvFTD have greater functional impairment when compared to those with other subtypes of frontotemporal lobar degeneration or Alzheimer's disease. The studies documented a significant association between cognitive impairment and measures of functionality in these patients. In addition, it has been suggested that the cognitive profile of patients may predict faster functional decline. The selected studies are listed in Table 1, with their respective objectives, samples, cognitive and functional measures, and results.

\section{Discussion}

The aim of the present study was to conduct a systematic review of studies investigating the functional profile of patients with bvFTD. A total of 14 clinical studies were identified whose aims were predominantly to compare the cognitive and functional performances of patients with bvFTD against other dementia types; provide a description of the functional profile of FTLD subtypes, comparing them with each other and noting which types presented greatest functional and cognitive decline; investigate the predictors of functional decline in bvFTD; compare indirect and direct functional performance of patients for different dementia subtypes; and assess scales for staging and measuring neuropsychiatric, dysexecutive, and functional symptoms in bvFTD.

The findings of the studies included in this review are in line with earlier reports indicating that patients with bvFTD are younger than patients with other dementias given that early onset and rapid progression are frequent, particularly with regard to the bvFTD subtype [10]. Among the studies reviewed (see Table 1), patient age ranged from 50 to 60 years.

Relations between Functional Impairment and Cognition. Some studies included in this review suggested a significant association between cognitive and functional performance in patients with bvFTD. Razani et al. [8], for instance, reported that impaired verbal fluency was a predictor of functional incapacity, assessed directly by the DAFS-R and that performance on the WCST was moderately correlated with IADLs as reported by caregivers. Piquard et al. [7] found that worse performance on the MMSE was associated with functional incapacity, and that higher score on the CDR was a predictor of functional decline in patients with bvFTD. Additionally, Josephs et al. [2] reported that poor performance on executive, visuospatial, and language functions were indicative of more rapid progression in functional activities. These findings point to a relationship between cognitive and functional performance among patients with bvFTD that is not restricted to changes in executive functions. In other words, decline in other functions also appears to contribute to the functional deficits in this condition. In disagreement with the results outlined above, Mioshi et al. [9] failed to find any significant association between performance on the ACE-R and the DAD.

Studies Comparing Functional Impairment across Dementia Types. The studies reviewed suggested that individuals with bvFTD present greater functional impairment compared to patients with other FTLD subtypes or AD. Using the DAD, 


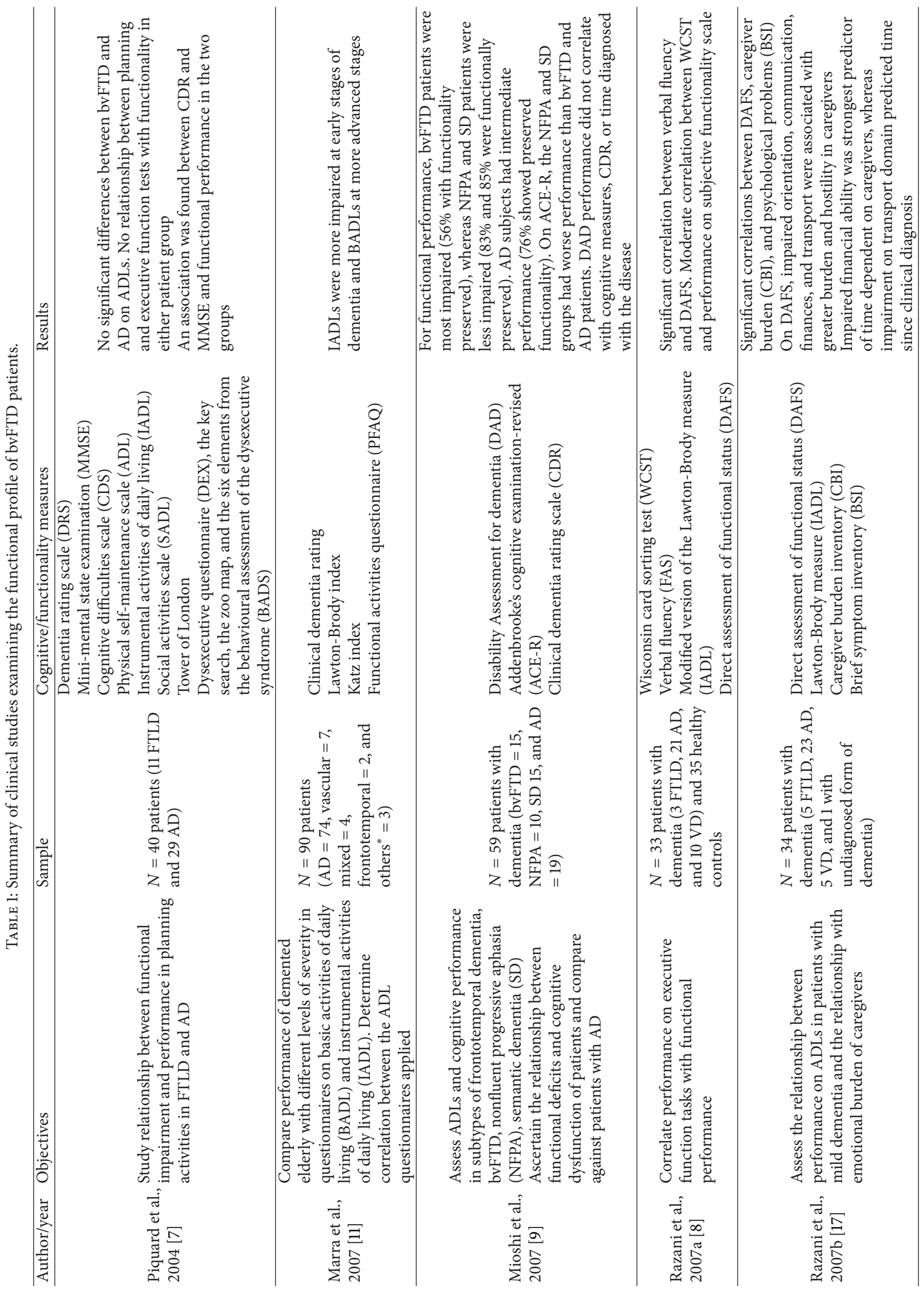




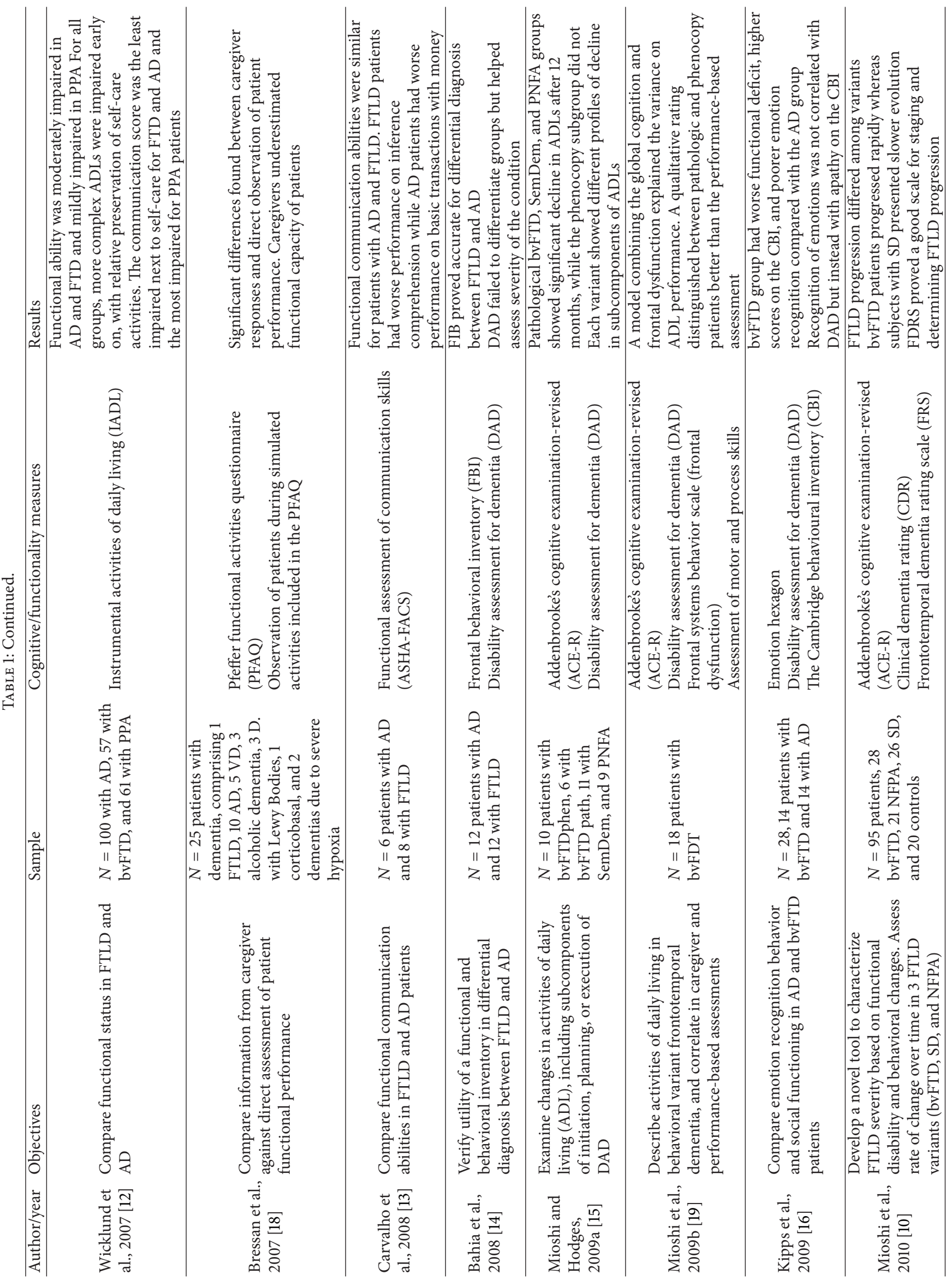




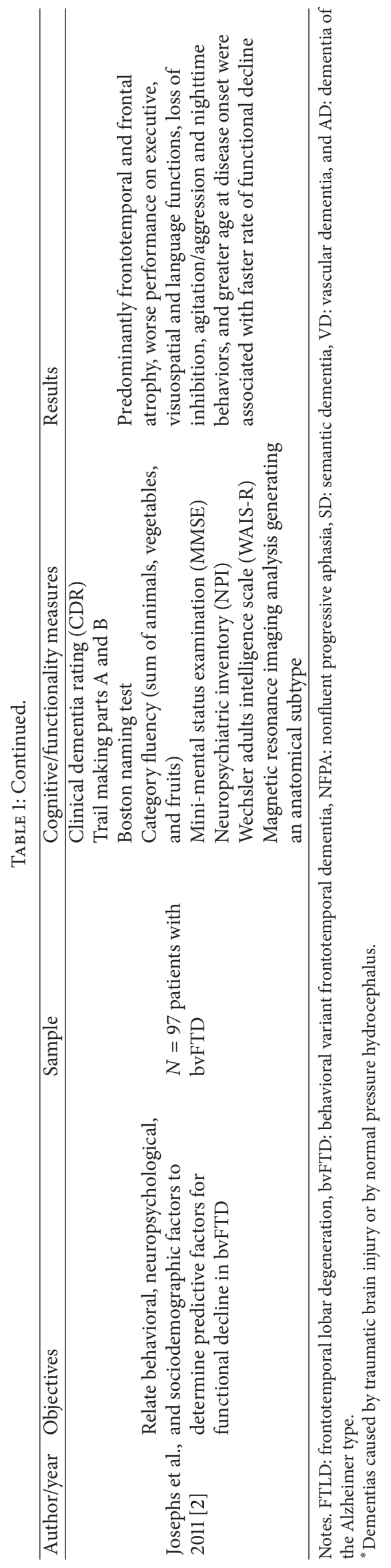


Mioshi et al. [9] showed that patients with bvFTD had poorer functional performance than patients with SD, NFPA or AD. This group had lower scores even on BADLs such as getting dressed, feeding, and hygiene. For IADLs, the worst performances were seen in finances, correspondence, and leaving the home. Poorer performances were also evident for the use of the telephone, domestic and leisure time activities, managing medications, and preparing meals, besides the impairments observed in $\mathrm{AD}$ patients. The authors highlighted the devastating impact of functional changes on the everyday routine of patients with bvFTD and the burden placed on their caregivers. These results were confirmed in later studies by the same group $[15,16]$.

Mioshi and Hodges [15] aimed to examine changes in activities of daily living (ADL), including subcomponents of the DAD and level of functional impairment in the FRS. The patients were subdivided into bvFTD pathological and phenocopy subgroups (patients who met diagnostic criteria but the disease did not progress), SD, and PNFA. The results indicated that pathological bvFTD, SD and PNFA groups showed significant decline in ADLs after 12 months, while the phenocopy subgroup did not. According to the FRS, the variants showed different levels of functional impairment. Virtually, all patients with bvFTD fell within the moderate, severe, very severe, or profound categories, whereas for the PNFA and SD groups, 25\% fell within the mild category and there were no patients regarded as very severe or profound. The proportion of patients with PNFA in the severe category was about the double in comparison to patients with SD. In general, results suggested patients with bvFTD were more dependent on BADLs and IADLs than the other variants. In addition, functional performance and cognitive scores were significantly correlated and FTD variants showed differential annual rates of functional decline, with faster decline among bvFTD patients.

The study by Wicklund et al. [12] examined the functional profile of patients diagnosed with $\mathrm{AD}$, bvFTD, and primary progressive aphasia (PPA). Results showed that functional ability was moderately impaired in $\mathrm{AD}$ and bvFTD and mildly impaired in PPA. Self-care activities were the least impaired in all groups, whereas more complex ADLs, such as shopping and management of finances, were impaired early on. Communication ability was the least impaired, next to self-care for bvFTD and AD and the most impaired for PPA patients.

Two studies failed to find differences among patients diagnosed with FTLD and AD on assessments of functionality. Carvalho et al. [13] found no significant difference in functional communication between $\mathrm{AD}$ and FTLD patients. Similarly, Bahia et al. [14] found no significant differences between patients with $\mathrm{AD}$ and FTLD on DAD scores. In their sample, DAD did not prove accurate for differential diagnosis of these dementia types. These negative results may be ascribed to the small sample size or to the fact that sample could not be stratified into FTLD subtypes.

Two of the reviewed studies examined functional performance of FTLD patients with direct assessment, involving the observation of the patient during functional activities. Razani et al. [17] found significant correlations between poor performance on the DAFS and increased burden and psychological distress of caregivers. Impaired performance on orientation, communication, finances and transport were associated with greater burden and hostility in caregivers. Comparing direct and indirect assessments of functionality in a mixed cohort of dementia patients, only one of which had FTLD, Bressan et al. [18] noted discordance between the opinion of caregivers/family members and actual observed performance of the patient, whereby informants often underestimated patients' potential to perform ADLs.

The reviewed studies highlighted the importance of carrying out functional assessment of patients with suspected bvFTD given the relevance of these changes for the diagnosis and clinical management of this dementia subtype. Some authors, such as Razani et al. [8], highlighted the dysexecutive profile of these patients and the significant impact on performing ADLs. Patients in the cited study exhibited difficulties starting tasks, estimating time, switching between tasks, and dealing with tasks of differing priorities. Also, subjects had problems controlling their impulses and exhibited deficits in chronological ordering, impatience, and emotional lability, directly impacting the performance of ADL. It is widely accepted that cognitive changes explain the functional deficits seen in patients with bvFTD. However, authors such as Mioshi et al. [15] suggested that apathy and impulsiveness, typical among this patient group, may also influence the functional deficits observed.

Notably, some studies suggested that patients with bvFTD had lower survival rate compared to patients with $\mathrm{AD}$, possibly associated with greater functional impairment [10]. Studies involving scales of severity and staging of bvFTD, such as the investigations by Marra et al. [11], Mioshi et al. [10], and Josephs et al. [2], support the hypothesis that the severity of bvFTD, together with its cognitive and neuropsychiatric symptoms, may modulate functional performance.

The present work has limitations including the paucity of studies identified investigating the functional profile of patients with bvFTD. Additionally, some of the reviewed studies included a very small number of patients diagnosed with bvFTD, and this fact may render them more informative about dementia in general than bvFTD. It is noteworthy that the vast majority of studies in the current literature have investigated the functional profile of $\mathrm{AD}$ patients, healthy controls, and elderly with mild cognitive impairment [1924]; therefore, the functional status of patients with FTLD requires further investigation. Future clinical and epidemiologic studies on FTLD should include instruments assessing functional performance in order to better characterize the patient profile. Such studies should have an impact on rehabilitation strategies geared towards patients with FTLD. Such findings should be relevant for the planning and delivery of support for families and may reinforce the need for tailored caregiver support that should take into account the disease stage and the functional limitations of the patient.

\section{Acknowledgment}

This research was funded by Fundação de Amparo à Pesquisa do Estado de São Paulo, Grant no. 11/04804-1. 


\section{References}

[1] O. Piguet, M. Hornberger, B. P. Shelley, C. M. Kipps, and J. R. Hodges, "Sensitivity of current criteria for the diagnosis of behavioral variant frontotemporal dementia," Neurology, vol. 72, no. 8, pp. 732-737, 2009.

[2] K. A. Josephs Jr., J. L. Whitwell, S. D. Weigand et al., "Predicting functional decline in behavioural variant frontotemporal dementia," Brain, vol. 134, no. 2, pp. 432-448, 2011.

[3] I. R. A. MacKenzie, M. Neumann, E. H. Bigio et al., "Nomenclature and nosology for neuropathologic subtypes of frontotemporal lobar degeneration: an update," Acta Neuropathologica, vol. 119, no. 1, pp. 1-4, 2010.

[4] I. R. A. Mackenzie, M. Neumann, E. H. Bigio et al., "Nomenclature for neuropathologic subtypes of frontotemporal lobar degeneration: consensus recommendations," Acta Neuropathologica, vol. 117, no. 1, pp. 15-18, 2009.

[5] M. F. Mendez, J. S. Shapira, A. McMurtray, E. Licht, and B. L. Miller, "Accuracy of the clinical evaluation for frontotemporal dementia," Archives of Neurology, vol. 64, no. 6, pp. 830-835, 2007.

[6] K. Rascovsky, J. R. Hodges, D. Knopman et al., "Sensitivity of revised diagnostic criteria for the behavioural variant of frontotemporal dementia," Brain, vol. 134, no. 9, pp. 2456-2477, 2011.

[7] A. Piquard, C. Derouesné, L. Lacomblez, and E. Siéroff, "Planning and activities of daily living in Alzheimer's disease and frontotemporal dementia," Psychol Neuropsychiatr Vieil, vol. 2, no. 2, pp. 147-156, 2004.

[8] J. Razani, R. Casas, J. T. Wong, P. Lu, C. Alessi, and K. Josephson, "Relationship between executive functioning and activities of daily living in patients with relatively mild dementia," Applied Neuropsychology, vol. 14, no. 3, pp. 208-214, 2007.

[9] E. Mioshi, C. M. Kipps, K. Dawson, J. Mitchell, A. Graham, and J. R. Hodges, "Activities of daily living in frontotemporal dementia and Alzheimer disease," Neurology, vol. 68, no. 24, pp. 2077-2084, 2007.

[10] E. Mioshi, S. Hsieh, S. Savage, M. Hornberger, and J. R. Hodges, "Clinical staging and disease progression in frontotemporal dementia," Neurology, vol. 74, no. 18, pp. 1591-1597, 2010.

[11] T. A. Marra, L. S. M. Pereira, C. D. C. M. Faria, D. S. Pereira, M. A. A. Martins, and M. G. A. Tirado, "Avaliação das atividades de vida diária de idosos com diferentes níveis de demência (Evaluation of the activities of daily living of elderly people with diferente levels of dementia)," Revista Brasileira de Fisioterapia, vol. 11, no. 4, pp. 267-273, 2007.

[12] A. H. Wicklund, N. Johnson, A. Rademaker, B. B. Weitner, and S. Weintraub, "Profiles of decline in activities of daily living in non-Alzheimer dementia," Alzheimer Disease and Associated Disorders, vol. 21, no. 1, pp. 8-13, 2007.

[13] I. A. M. Carvalho, V. S. Bahia, and L. L. Mansur, "Functional communication ability in frontotemporal lobar degeneration and Alzheimer's disease," Dementia e Neuropsychologia, vol. 2, no. 1, pp. 31-36, 2008.

[14] V. S. Bahia, M. N. Maia-Silva, R. Viana et al., "Behavioral and activities of daily living inventories in the diagnosis of frontotemporal lobar degeneration and Alzheimer's disease," Dementia e Neuropsychologia, vol. 2, no. 2, pp. 108-113, 2008.

[15] E. Mioshi and J. R. Hodges, "Rate of change of functional abilities in frontotemporal dementia," Dementia and Geriatric Cognitive Disorders, vol. 28, no. 5, pp. 419-426, 2009.
[16] C. M. Kipps, E. Mioshi, and J. R. Hodges, "Emotion, social functioning and activities of daily living in frontotemporal dementia," Neurocase, vol. 15, pp. 182-189, 2009.

[17] J. Razani, B. Kakos, C. Orieta-Barbalace et al., "Predicting caregiver burden from daily functional abilities of patients with mild dementia," Journal of the American Geriatrics Society, vol. 55, no. 9, pp. 1415-1420, 2007.

[18] L. A. Bressan, F. A. C. Vale, and J. G. Speciali, "The daily life of patients with dementia, a comparative study between the information provided by the care giver and direct patient assessment," Dementia e Neuropsychologia, vol. 1, no. 3, pp. 288295, 2007.

[19] E. Mioshi, C. M. Kipps, and J. R. Hodges, "Activities of daily living in behavioral variant frontotemporal dementia: differences in caregiver and performance-based assessments," Alzheimer Disease and Associated Disorders, vol. 23, no. 1, pp. 70-76, 2009.

[20] M. E. Converso and L. Iartelli, "Caracterização e análise do estado mental e funcional de idosos institucionalizados em instituições públicas de longa permanência," Jornal Brasileiro de Psiquiatria, vol. 56, no. 4, pp. 448-454, 2007.

[21] F. S. Pereira, M. S. Yassuda, A. M. Oliveira, and O. V. Forlenza, "Executive dysfunction correlates with impaired functional status in older adults with varying degrees of cognitive impairment," International Psychogeriatrics, vol. 20, no. 6, pp. 11041115, 2008.

[22] F. S. Pereira, A. M. Oliveira, B. S. Diniz, O. V. Forlenza, and M. S. Yassuda, "Cross-cultural adaptation, reliability and validity of the DAFS-R in a sample of Brazilian older adults," Archives of Clinical Neuropsychology, vol. 25, no. 4, pp. 335-343, 2010.

[23] F. S. Pereira, M. S. Yassuda, A. M. Oliveira et al., "Profiles of functional deficits in mild cognitive impairment and dementia: benefits from objective measurement," Journal of the International Neuropsychological Society, vol. 16, no. 2, pp. 297-305, 2010.

[24] E. C. Costa, A. Y. K. Nakatani, and M. M. Bachioni, "Capacidade de idosos da comunidade para desenvolver atividades de vida diária e atividades instrumentais de vida diária," Acta Paulista de Enfermagem, vol. 19, no. 1, pp. 43-48, 2006. 


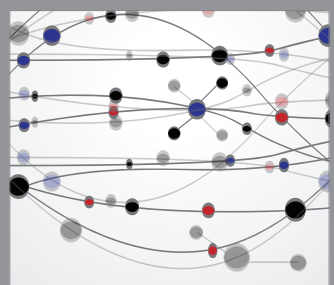

The Scientific World Journal
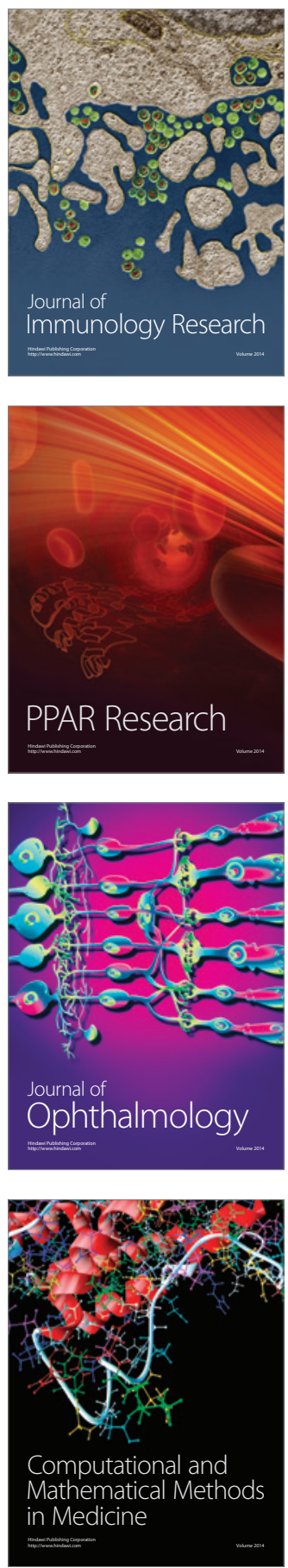

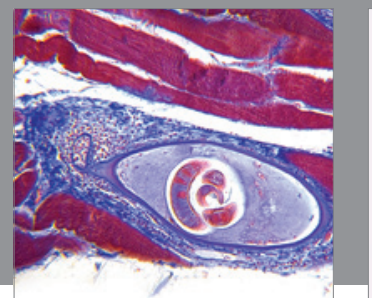

Gastroenterology

Research and Practice
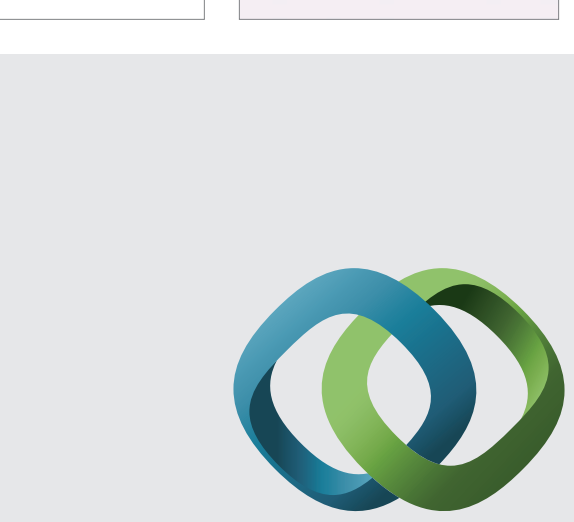

\section{Hindawi}

Submit your manuscripts at

http://www.hindawi.com
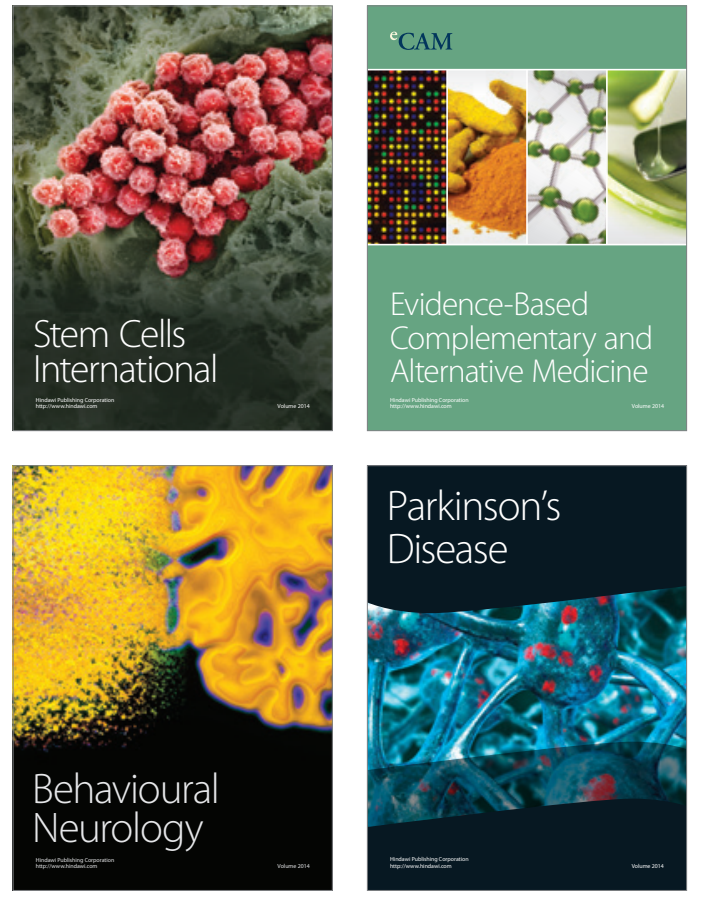
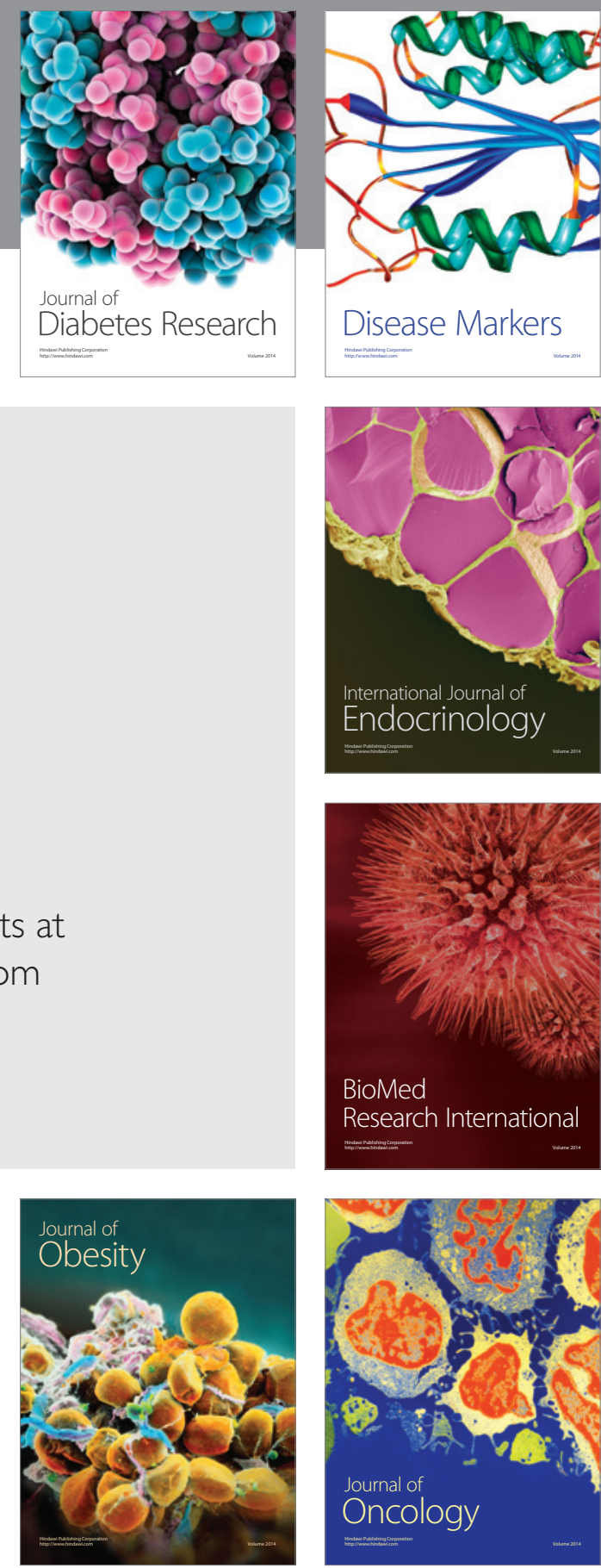

Disease Markers
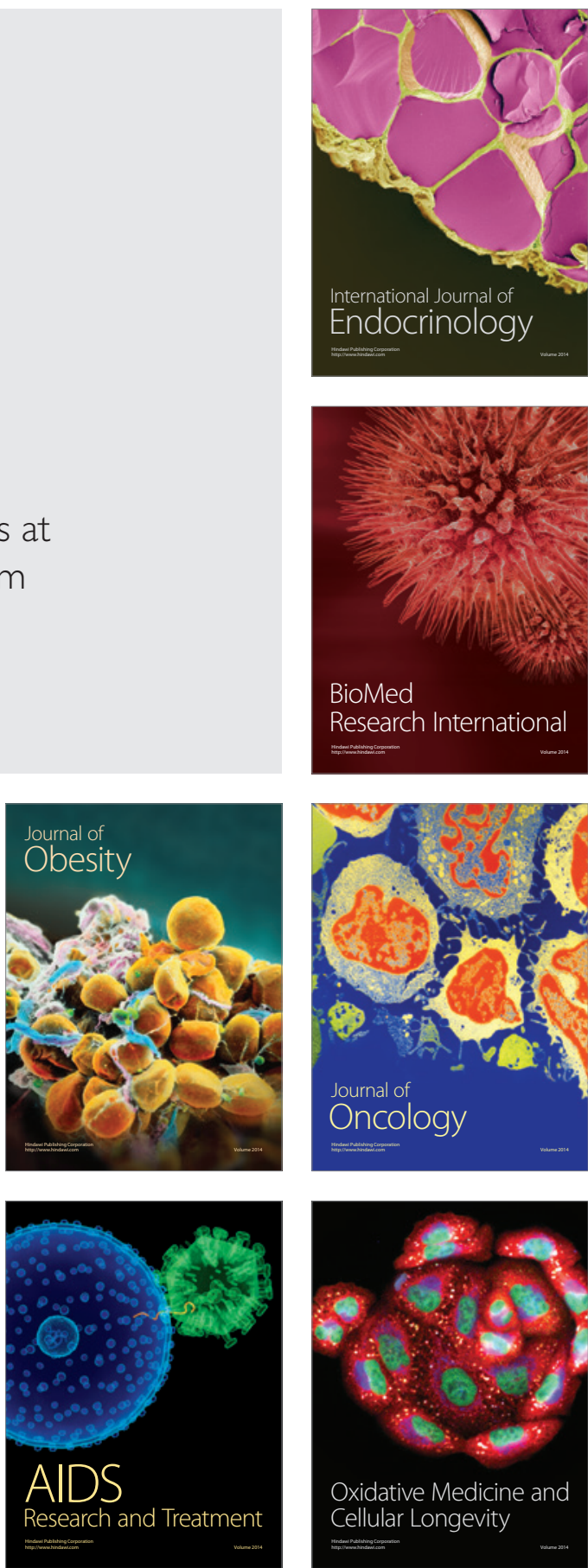